\title{
Analysis and Design of Dirty Paper Coding by Transformation of Noise
}

\author{
Young-Seung Lee and Sae-Young Chung \\ Department of Electrical Engineering and Computer Science \\ Korea Advanced Institute of Science and Technology \\ E-mail: sychung@ee.kaist.ac.kr
}

\begin{abstract}
We design a coding scheme for Costa's dirty paper coding (DPC) [6] using a channel and a shaping code. We show that by transforming the channel noise distribution the DPC channel can be converted into the binary erasure channel (BEC) with binary interference with memory. Furthremore, the messages exchanged during the iterative decoding between the channel and shaping codes become one dimensional under the new model. We analyze the iterative decoding and find good shaping and channel code pairs using some closed-form extrinsic information transfer (EXIT) curves. We verify that our dirty paper codes designed using this method are also good for the original DPC channel with the additive white Gaussian noise (AWGN) and arbitrary interference.

Our implementation of DPC uses short block codes such as repetition codes for shaping codes. Although the shaping gains of such codes are not very high, they may provide a better complexity-performance trade-off for simple practical implementations of DPC. Furthermore, we show accurate theoretical analysis is possible for such codes under our channel model.
\end{abstract}

\section{INTRODUCTION}

DPC by Costa [6] can achieve the AWGN capacity in the presence of interference as long as the interference is noncausally known at the transmitter. One practical importance is that it can achieve the capacity of vector Gaussian broadcast channels [2]. However, the proof method in [6] was information theoretic and its practical implementation has been unrealized for a long time.

Recently, there have been some practical implementations of DPC that can achieve the capacity very closely using nested lattices and more general superposition coding between shaping and channel codes [7], [8], [9]. The shaping code is typically implemented using a convolutional code. The complexity of DPC, however, is high due to the complexity of convolutional codes especially when a good shaping gain is needed. In this paper, we use short block codes such as repetition codes as shaping codes. Although the shaping gains of such codes are not very high (up to about 0.47 ), they may provide a better complexityperformance trade-off for simple practical implementations of DPC. Furthermore, we show accurate theoretical analysis is possible for such codes due to their simplicity, which can provide us some intuition on how to design good channel and shaping code pairs for DPC.
By transforming the channel noise distribution to a simpler one, the messages exchanged during the iterative decoding between the channel and shaping codes corresponding to the transformed noise become one dimensional. We show the transformed channel is equivalent to the BEC with binary interference from the shaping code. Note that the effect of original continuous-time interference in the DPC channel is eliminated at the transmitter, but the shaping code is still interfering with the channel code during the decoding. Closed-form EXIT [3], [4] curves exist for the shaping and channel codes we consider (we use low-density parity-check codes for the channel code) since the codes are simple and since the converted channel is simple. Since codes design for one channel are often good for some other channels, it motivates our approach of converting the channel. We indeed verify that the codes designed assuming the transformed noise are also good for the original AWGN channel.

The paper is organized as follows. Section II reviews our implementation of the DPC and the noise transformation. Section III shows analysis and simulation results of our designed codes. We conclude our paper in Section IV.

\section{SySTEM MODEL}

\section{A. Dirty paper coding}

The following is our channel model

$$
Y=X+S+Z,
$$

where $X$ is the transmitted signal with power constraint $E\left[X^{2}\right] \leq P_{X}, S$ is the interference non-causally known only at the transmitter with an arbitrary distribution, $Z$ is the AWGN with variance $P_{Z}$, and $Y$ is the received signal. The DPC can achieve $\frac{1}{2} \log \left(1+\frac{P_{X}}{P_{Z}}\right)$ [6], which is same as the capacity of the AWGN channel without any interference, i.e., $Y=X+Z$.

\section{B. Transmitter and receiver}

Our implementation of DPC is based on the superposition of channel and shaping codes [9] and closely resembles the one by Erez and ten Brink [7]. Fig. 1 shows the transmitter, where $k_{c}$ information bits are encoded into $n_{c}$ bits by a strong channel code such as turbo or low-density paritycheck (LDPC) codes with a long block length. Let $W$ denote 
the encoded binary sequence of $n_{c}$ bits. We assume BPSK signaling where 0 and 1 are mapped to 0 and 1 , respectively. We use a random interleaver between the channel and the shaping codes if for example the turbo code is used for the channel code to randomize the interface between the two codes. Note that the random interleaver is not needed if the channel code is LDPC since neighbor variables in the LDPC code do not necessarily have a short distance in the LDPC graph.

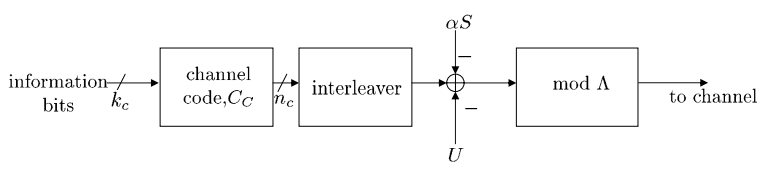

Fig. 1. Transmitter

Non-causally known interference $S$ and a random dither $U$ are subtracted from encoded sequence, where $S$ is multiplied by the minimum mean square error (MMSE) scaling factor $\alpha=P_{X} /\left(P_{X}+P_{Z}\right)$. The shaping code is implemented as a lattice $\Lambda$ using the construction A using a binary linear block code. The shaping code performs a modulo operation mod $\Lambda$, which is implemented using the Viterbi algorithm. We assume the dimension of the shaping lattice $\Lambda$ is equal to $n_{c}$. More generally the dimension of $\Lambda$ can be smaller than $n_{c}$ as long as it divides $n_{c}$ assuming the shapping lattice is applied many times per one encoded sequence $W$. Let $X$ denote the transmitted signal in Fig. 1. Then the relationship between the encoded sequence $W$ and the transmitted sequence $X$ is given by

$$
X=(W-\alpha S-U) \bmod \Lambda .
$$

Fig. 2 shows an equivalent model for the transmitter, where the outputs of the two codes, the channel and the shaping codes, are XOR'ed. The input to the shaping code can be considered as a virtual input minimizing the transmission power after $\bmod 2 \mathbb{Z}^{n_{c}}$, where $2 \mathbb{Z}^{n_{c}}$ is the $n_{c}$-dimensional integer lattice scaled by 2 . The equivalence of the two schemes can be seen from the lattice chain $\mathbb{Z}^{n_{c}} / \Lambda / 2 \mathbb{Z}^{n_{c}}$ [7]. The equivalent model in Fig. 2 is helpful for designing the decoder since the decoder can perform an iterative decoding between the shaping and the channel codes to recover the original message as can be seen from Fig. 2.

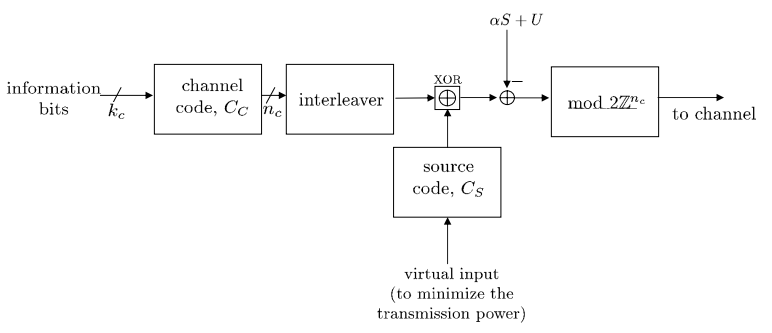

Fig. 2. Equivalent transmitter using superposition of two codes in $G F(2)$

The interference $S$ and the noise $Z$ are then added to the transmitted signal $X$ and the receiver receives $Y=$ $X+S+Z$. Then the MMSE scaling $\alpha=P_{x} /\left(P_{x}+P_{z}\right)$ is applied, the random dither $U$ is added back, and $\bmod \Lambda$ is performed to get $Y^{\prime}=\alpha Y+U \bmod \Lambda$. Using the inflated lattice lemma [1], the resulting signal $Y^{\prime}$ can be equivalently written as

$$
Y^{\prime}=\left(W+Z^{\prime}\right) \bmod \Lambda,
$$

where $Z^{\prime}=[(\alpha-1) U+\alpha Z] \bmod \Lambda$ is the effective noise. This effective noise will behave roughly as Gaussian at high SNR and as a uniform distribution at low SNR. Note that the exact characterization of this noise for arbitrary SNR is difficult if the dimension of the shaping lattice is high.

\section{Transformation of noise}

We then transform the effective noise $Z^{\prime}$ into $Z^{\prime \prime}$, where the $i$-th element $Z_{i}^{\prime \prime}$ of $Z^{\prime \prime}$ is given by

$$
Z_{i}^{\prime \prime}= \begin{cases}0 & \text { with probability } 1-\epsilon \\ R_{i} & \text { with probability } \epsilon\end{cases}
$$

where $R_{i}$ for $1 \leq i \leq n_{c}$ is uniform in $[-1,1)$ that is independent from $W, S, Z$, and $\left\{R_{j} \mid j \neq i\right\}$ and $0 \leq \epsilon \leq 1$ is a function of $\alpha$ and the noise variance of the original AWGN so that the capacity remains the same.

Assuming this new noise distribution, the signal $Y^{\prime}$ can be rewritten as

$$
Y^{\prime}=\left[\left(W_{c} \oplus W_{s}\right)+Z^{\prime \prime}\right] \bmod 2 \mathbb{Z}^{n_{c}},
$$

where $W_{c}$ is the $n_{c}$-bit binary sequence from the channel encoder and $W_{s}$ is $n_{c}$-bit the binary sequence from the source encoder and $\oplus$ is the XOR operation. Since $Z_{i}^{\prime \prime}$ will completely destroy any useful information in the $i$-th element $Y_{i}^{\prime}$ of $Y^{\prime}$ with probability $\epsilon$, we have the following equivalent information at the receiver

$$
\left(W_{c} \oplus W_{s}\right) \cdot T
$$

where - is the multiplication in $G F(2)$ and $T$ is an i.i.d. binary sequence independent of $W_{c}$ and $W_{s}$, where each element is 0 with probability $\epsilon$. Therefore, the transformed channel is now equivalent to the BEC with an additive binary interference from $W_{s}$.

By using this channel model, we can not only simplify analysis but can also have an accurate prediction of the behavior of shaping and channel codes for the original DPC channel due to the similarity of the behavior of the iterative decoding between the BEC and the AWGN channels.

\section{ANALYSIS AND DESIGN}

In this section, we show our main analysis and design of DPC codes using the system model described in the previous section.

\section{A. Shaping gain of repetition code}

Let $|\nu|$ denote the volume of the fundamental Voronoi region $\nu$ of $\Lambda$. The per-dimension second moment of the lattice $\Lambda$ is:

$$
P(\Lambda)=\frac{1}{N|\nu|} \int_{\nu}\|X\|^{2} d X
$$


where $N$ is the dimension of the lattice. The normalized second moment of the lattice $\Lambda$ is given by

$$
G(\Lambda)=\frac{P(\Lambda)}{|\nu|^{2 / N}}
$$

The shaping gain $g_{s}(\Lambda)$ is defined as the ratio between the normalized second moment of the integer lattice and $G(\Lambda)$.

$$
g_{s}(\Lambda)=\frac{\int_{-1 / 2}^{1 / 2} x^{2} d x}{G(\Lambda)}=\frac{1}{12 G(\Lambda)}
$$

Fig. 3 shows the shaping gain of the $(N, 1)$ repetition code, which is maximized for $N=8 \sim 12$ giving about $0.47 \mathrm{~dB}$. Note that the maximum possible shaping gain is

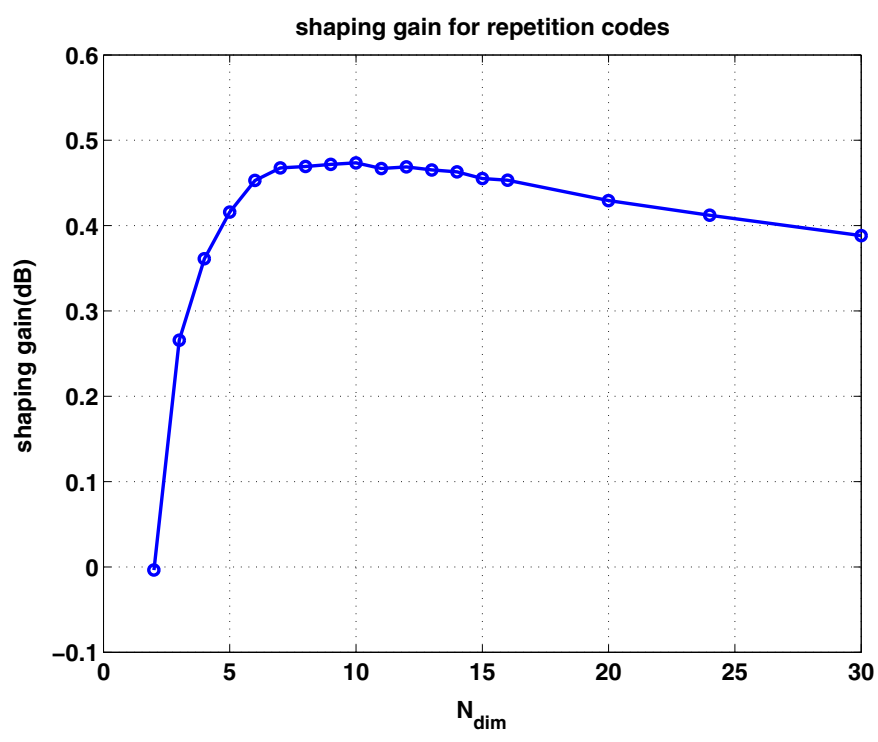

Fig. 3. Shaping gain of repetition coding

less than $0.8 \mathrm{~dB}$ even for a uniform $N$-sphere if $N$ is less than 8 . As a comparison, the shaping gains of some rate- $1 / 2$ convolutional codes are about $0.98,1.03,1.28 \mathrm{~dB}$ for paritycheck polynomials $\left(5_{8}, 7_{8}\right),\left(15_{8}, 17_{8}\right),\left(0561_{8}, 0753_{8}\right)$, respectively when $N>10^{3}$.

Repetition code has less shaping gain than more complicated convolutional codes, but it makes the implementation of DPC very simple, which can be desirable for some applications. We will mainly focus on $N$ between 8 and 12 since the shaping gain is maximized in the range. Since $N$ is small, we need to use many blocks of the repetition code to match the size of the channel code.

\section{B. The capacity seen by the channel code}

Fig. 4 shows the superposition of $X \in[0,1]^{N-1}$, which is a subset of encoded bits from the channel code and the codeword of the $(N, 1)$ repetition code, where one virtual bit $V$ is repeated $N$ times. The channel input is given by $[V \mid X \oplus V] \in[0,1]^{N}$. Each bit is independently erased with probability $\epsilon$ and received as $Y \in[0,1, E]^{N}$, where $E$ indicates erasure. Note that the virtual bit is treated as interference with equal probabilities between 0 and 1, i.e.,

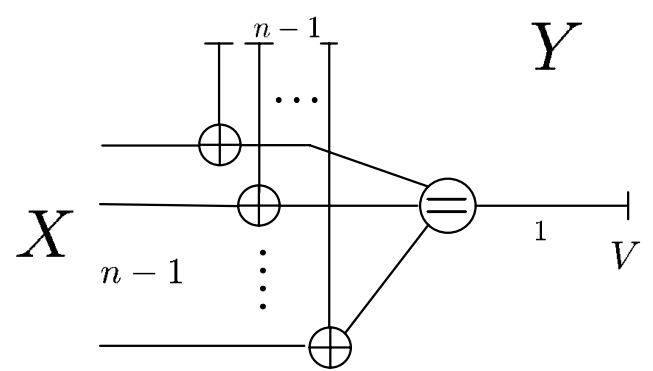

Fig. 4. Equivalent channel

$\operatorname{Pr}(V=0)=\operatorname{Pr}(V=1)=\frac{1}{2}$. The channel capacity seen by $X$ is given by

$$
C_{\epsilon}=I(X ; Y)=H(Y)-H(Y \mid X)
$$

By symmetry, we get $H(Y \mid X)=H(Y \mid X=0)$. Let $I$ denote a random variable indicating the number of erasures, i.e., $\operatorname{Pr}(I=i)=\left(\begin{array}{c}N \\ i\end{array}\right) \epsilon^{i}(1-\epsilon)^{N-i}$. Using this, (1) becomes:

$$
\begin{aligned}
C_{\epsilon} & =\frac{1}{N}\left\{-\sum_{i=0}^{N} \operatorname{Pr}(I=i)\left[\log _{2} \frac{\operatorname{Pr}(I=i)}{\left(\begin{array}{c}
N \\
i
\end{array}\right)}+(i-N)\right]\right. \\
& \left.+\sum_{i=0}^{N-1} \operatorname{Pr}(I=i) \log _{2} \frac{\operatorname{Pr}(I=i)}{2\left(\begin{array}{c}
N \\
i
\end{array}\right)}+\epsilon^{N} \log _{2}\left(\epsilon^{N}\right)\right\},
\end{aligned}
$$

which can be simplified as follows:

$C_{\epsilon}=\frac{1}{N}\left[N(1-\epsilon)-\left(1-\epsilon^{N}\right)\right]=(1-\epsilon)-\frac{1-\epsilon^{N}}{N}$.

As expected, we can see that when $N$ tends to infinity, the rate penalty due to the shaping code tends to zero. Fig. 5 shows the capacity of the BEC with binary interference from the shaping code for various values of $N$.

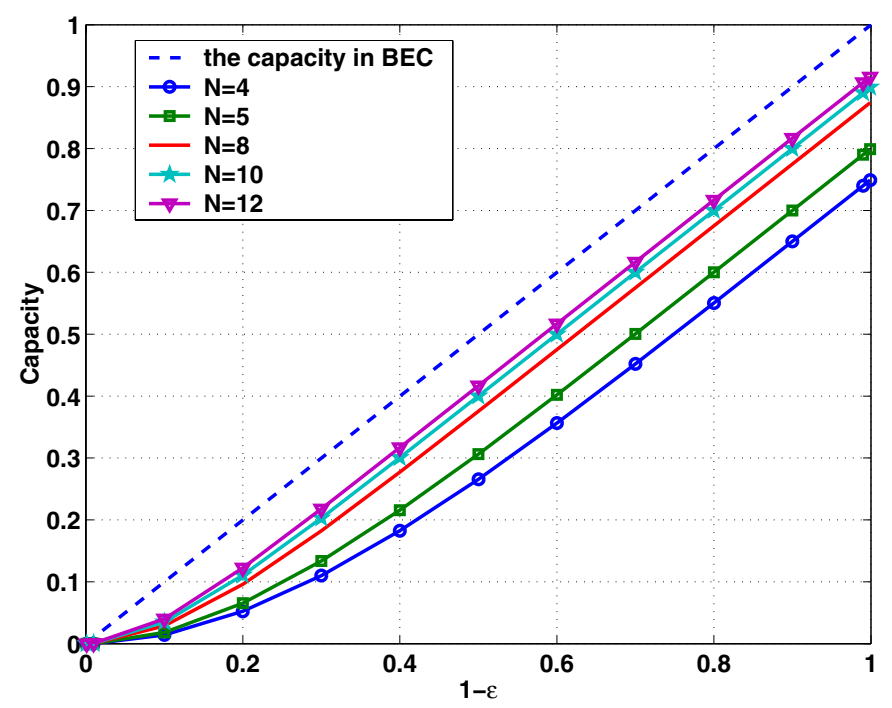

Fig. 5. Capacity of BEC with interference from repetition code 


\section{EXIT curve for the shaping code}

Fig. 6 shows extrinsic information exchanges at the receiver assuming an LDPC code is used as the channel code, where $I_{A 1}, I_{A 2}, I_{E 1}$, and $I_{E 2}$ are messages to the channel code, to the shaping code, from the channel code, and from the shaping code, respectively. $\Pi$ denotes the random interleaver between the shaping and the channel codes, which can be the identity mapping when the channel code is LDPC as discussed before.

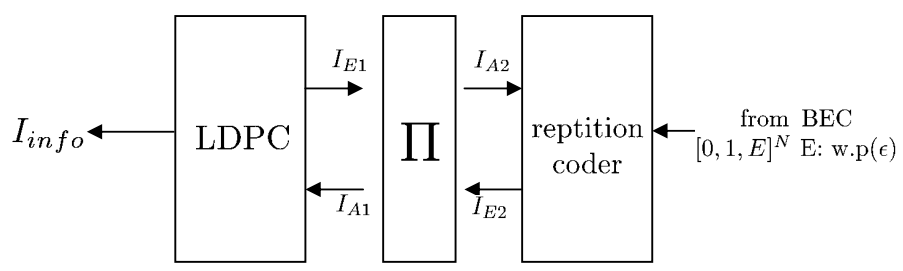

Fig. 6. Exchange of extrinsic information

The extrinsic information $I_{E 2}$ from the repetition code is given by:

$$
I_{E 2}=(1-\epsilon)\left\{1-\epsilon[1-(1-p)(1-\epsilon)]^{N-2}\right\}
$$

where $\epsilon$ is the erasure probability from the channel, $p$ is the erasure probability of the messages from the channel code, which is same as $1-I_{A 2}$.

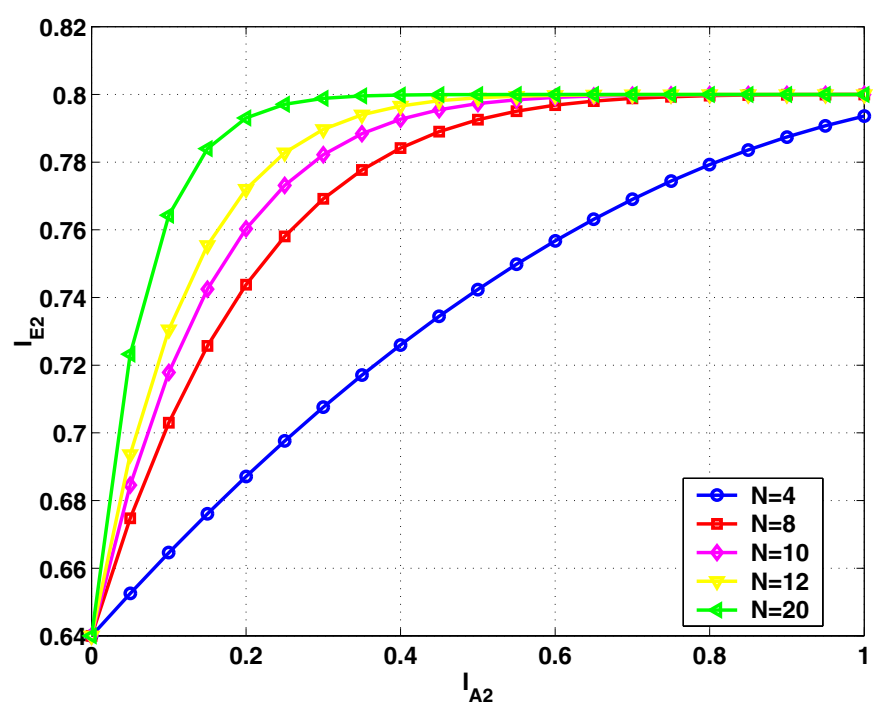

Fig. 7. EXIT curves for some shaping codes for $\epsilon=0.2$

Fig. 7 shows that $I_{E 2}$ will be saturated roughly to $I_{\text {channel }}=1-\epsilon$ as $I_{A 2}$ approaches 1 if $N$ is large, which can be verified in equation (4), i.e., when $p=0$ we get

$$
I_{E 2}=(1-\epsilon)\left(1-\epsilon^{N-1}\right) \simeq(1-\epsilon)=I_{\text {channel }} .
$$

This is because each bit of $X$ suffers from the channel noise as can be seen from Fig. 4.

\section{EXIT curve for LDPC codes}

A strong channel code is needed since the shaping code always has residual errors. In case of infinite-length irregular LDPC ensembles, a closed form EXIT curve is known [3], which is given as a parametric form as follows:

$$
\left(\frac{x}{\lambda(1-\rho(1-x))}, \Lambda(1-\rho(1-x))\right)
$$

where $x$ is a parameter, $\lambda(x)$ and $\rho(x)$ are degree polynomials of variable and check nodes, respectively, and $\Lambda(x)=$ $\frac{1}{\int_{0}^{1} \lambda} \int_{0}^{x} \lambda(y) d y$. For example, the $(3,6)$ regular LDPC code has $\lambda(x)=x^{2}, \rho(x)=x^{5}$, and $\Lambda(x)=x^{3}$. Note that this is an asymptotic result assuming infinite block length.

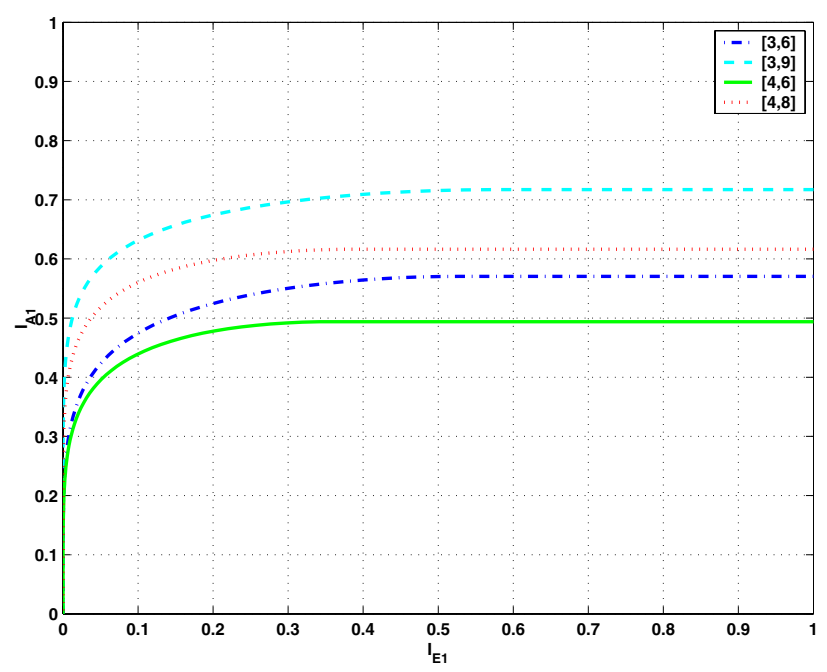

Fig. 8. EXIT curves for some LDPC code ensembles (axes are swapped)

We plot the EXIT curves for several regular LDPC ensembles in Fig. 8. Note that the shape of the curves in Fig. 7 and Fig. 8 are similar for large $N$. This means the gap between the EXIT curves for the shaping and channel codes can be made small by choosing appropriate shaping and channel code parameters. Note that since approaching the capacity of the BEC requires the flatness condition, we are motivated to reduce the EXIT gap between the two codes.

Fig. 9 shows the difference between the capacity seen by the channel code and the iterative decoding threshold computed from the EXIT curves of the two codes, where $N=10$ is assumed for the shaping code and regular LDPC codes with variable degree equal to 3 are assumed. The gap is less than about 0.12 and decreases as the check node degree is increased. Note that irregular codes should do better, but regular codes are not so bad especially when the rate of the channel code is high. In case of $\rho(x)=x^{300-1}$, the gap is only about 0.0253 .

\section{E. $A W G N$ and BEC}

Fig.10 shows EXIT curves for the shaping and the channel codes for the BEC and the AWGN channels, where the erasure probability is 0.3835 and the noise variance is 0.1844 . 


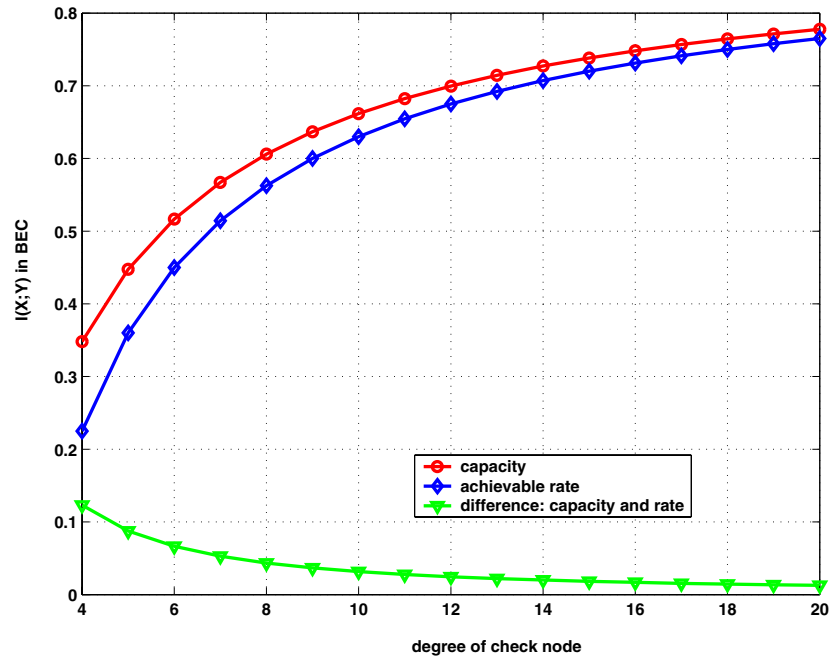

Fig. 9. Difference between capacity and the threshold of iterative decoding

It shows that the two channels give similar EXIT curves. Our DPC implementation is operating at SNR of $1.5 \mathrm{~dB}$, which is about $1.95 \mathrm{~dB}$ from the AWGN capacity. Considering a very low operating spectral efficiency and low shaping gain, the performance is reasonably good. We expect a better performance if an irregular LDPC code is used.

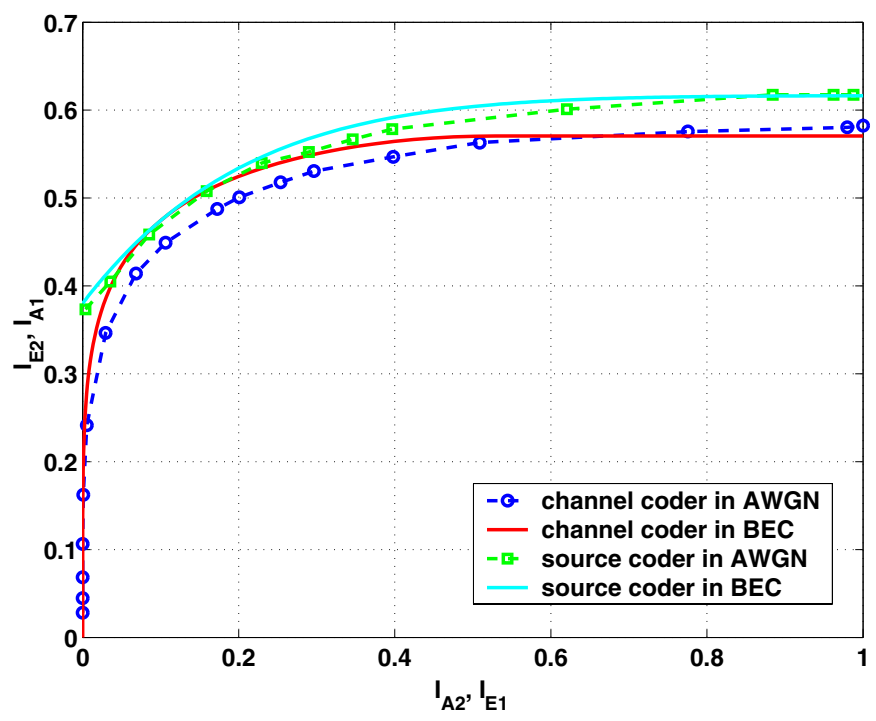

Fig. 10. EXIT curves for the BEC and the AWGN channels

\section{CONCLUSIONS}

We have considered an implementation of DPC using short block codes for shaping. We have shown that by an appropriate transform of the noise distribution we can convert the DPC channel into the BEC with a binary interference. Due to the simplicity of the shaping codes and our simplified channel model, closed-form analysis is possible for the iterative decoding between the channel and the shaping codes. We showed some regular LDPC codes are well matched to the shaping code in the EXIT chart when some low-rate repetition codes are used for shaping. We verified that our DPC codes are performing well for the original DPC channel.

\section{ACKNOWLEDGMENT}

This work was supported by the center for Broadband OFDM Mobile Access (BrOMA) at POSTECH through the ITRC program of the Korean MIC, supervised by IITA. (IITA-2006-C1090-0603-0037)

\section{REFERENCES}

[1] U. Erez, S. Shamai (Shitz), and R. Zamir, "Capacity and lattice strategies for cancelling known interference," Proc. IEEE ISITA, Honolulu, Hawaii, Nov. 2000, pp. 681-684.

[2] H. Weingarten, Y. Steinberg, and S. Shamai (Shitz), "The capacity region of the Gaussian MIMO broadcast channel," in Proc. IEEE Int Symp. Information Theory (ISIT), June 2004, pp. 174.

[3] C. Measson, A. Montanari and R. Urbanke, "Maxwell Construction: The Hidden Bridge between Iterative and Maximum a Posteriori Decoding," E-print: cs.IT/0506083. Submitted to IEEE Transactions on Information Theory.

[4] A. Ashikhmin, G. Kramer and S. ten Brink, "Extrinsic information transfer functions: model and erasure channel properties," IEEE Trans. on Information Theory, vol. 50, no. 11, pp. 2657- 2673, November 2004.

[5] T. Philosof, U. Erez, and R. Zamir. "Combined shaping and precoding for interference cancellation at low SNR," In Proc. of Int. Symp. Inform. Theory (ISIT2003), Yokohama, Japan, pp. 68 June 2003.

[6] M. H. M. Costa, "Writing on dirty paper," IEEE Trans. Inf. Theory, IT-29, pp. 439.441, May 1983.

[7] U. Erez and S. ten Brink, "A close-to-capacity dirty paper coding scheme," IEEE Trans. on Inform. Theory, October 2005.

[8] Y. Sun, A. Liveris, V. Stankovi, and Z. Xiong, "Near-capacity Dirtypaper Code Designs. Based on TCQ and IRA Codes," Proc. ISIT-2005 IEEE International Symposium on Information. Theory, Adelaide, Australia, September 2005.

[9] A. Bennatan, D. Burshtein, G. Caire, S. Shamai (Shitz), "Superposition coding for side-information channels," Information Theory, IEEE Transactions on, Vol. 52, No. 5.pp. 1872-1889. (2006). 